Pacific

Journal of

Mathematics

RIEMANNIAN MANIFOLDS ADMITTING ISOMETRIC IMMERSIONS BY THEIR FIRST EIGENFUNCTIONS

Ahmad El Soufi and Saïd Ilias 


\title{
RIEMANNIAN MANIFOLDS ADMITTING ISOMETRIC IMMERSIONS BY THEIR FIRST EIGENFUNCTIONS
}

\author{
Ahmad El Soufi ANd SaÏD Ilias
}

\begin{abstract}
Given a compact manifold $M$, we prove that every critical Riemannian metric $\mathrm{g}$ for the functional "first eigenvalue of the Laplacian" is $\lambda_{1}$-minimal (i.e., $(M, g)$ can be immersed isometrically in a sphere by its first eigenfunctions) and give a sufficient condition for a $\lambda_{1}$-minimal metric to be critical. In the second part, we consider the case where $M$ is the 2dimensional torus and prove that the flat metrics corresponding to square and equilateral lattices of $\mathbb{R}^{2}$ are the only $\lambda_{1}$ minimal and the only critical ones.
\end{abstract}

\section{Introduction.}

Many recent works concerning the spectrum of compact Riemannian manifolds have pointed out the importance of a particular class of Riemannian metrics which we called in [5] $\lambda_{1}$-minimal. Recall that a metric $g$ on a compact $m$-dimensional manifold $M$ is $\lambda_{1}$-minimal if the eigenspace $E_{1}(g)$ associated to the first nonzero eigenvalue $\lambda_{1}(g)$ of the Laplacian of $g$ contains a family $f_{1}, \ldots f_{k}$ of functions satisfying: $\sum_{1<i<k} d f_{i} \otimes d f_{i}=g$. It follows from a well known result of Takahashi [8] that this last condition is equivalent to the fact that the map $f=\left(f_{1}, \ldots f_{k}\right)$ is a minimal isometric immersion from $(M, g)$ into the Euclidean sphere $\mathbb{S}_{r}^{k-1}$ of radius $r=\sqrt{\frac{m}{\lambda_{1}(g)}}$.

The best known examples of $\lambda_{1}$-minimal metrics are the standard metrics of rank one compact symmetric spaces (i.e., spheres and projective spaces). More generally, any Riemannian irreducible homogeneous space is $\lambda_{1}$-minimal. Also, Yau [9] conjectured that a minimal embedded hypersurface of a Euclidean sphere, carrying the induced metric, must be $\lambda_{1}$-minimal.

In [2], Berger showed that the $\lambda_{1}$-minimality of a metric $g$ is strongly related to the extremality of $g$ for a spectral functional involving the $k$-smallest eigenvalues of the Laplacian (where $k$ is the multiplicity of $\lambda_{1}(g)$ ). Recently, Nadirashvili [7] considered the functional $\lambda_{1}: g \mapsto \lambda_{1}(g)$ defined on the set of Riemannian metrics of given area on a compact surface $M$ and showed that the extremal metrics of this functional are $\lambda_{1}$-minimal (here extremality is defined in a generalized sense because of the non-differentiability of $\left.\lambda_{1}\right)$. 
In the first part of this paper we generalize Nadirashvili's theorem to higher dimensions (Theorem 1.1). We also give a sufficient condition for a $\lambda_{1}$-minimal metric to be extremal for $\lambda_{1}$ (Proposition 1.1).

Using results established by us in [4] about $\lambda_{1}$-minimal metrics we deduce that (Corollary 1.1), if $g$ is an extremal metric of the $\lambda_{1}$ functional then:

(i) The multiplicity of $\lambda_{1}(g)$ is at least equal to $m+1$ and equality holds only for the standard metric of Euclidean spheres.

(ii) The restriction of the $\lambda_{1}$ functional to the conformal class of $g$ achieves its maximum at $g$. In particular, the $\lambda_{1}$ functional has no local minima.

(iii) The metric $g$ is, up to dilatation, the unique extremal metric in its conformal class.

(iv) If $g$ is not isometric to the standard metric of a Euclidean sphere then any conformal diffeomorphism of $(M, g)$ is an isometry.

The second part of this paper deals with the classification of $\lambda_{1}$-minimal metrics and of the extremal metrics of the $\lambda_{1}$ functional. The only manifold for which this classification was available is the 2-dimensional sphere. Indeed, on $\mathbb{S}^{2}$ the standard metric is (up to dilatation) the only one to be $\lambda_{1}$-minimal and the only extremal metric for $\lambda_{1}$ (this follows from the uniqueness of the conformal class on $\mathbb{S}^{2}$ and property (iii) above).

The main theorem of Section 2 (Theorem 2.1) states that in genus one (i.e., on the torus $\mathbf{T}^{2}$ ) there exists, up to dilatation, exactly two $\lambda_{1}$-minimal metrics: The Clifford metric $g_{c l}$ and the equilateral metric $g_{e q}$ induced from the Euclidean metric respectively on $\mathbb{R}^{2} / \mathbf{Z}^{2}$ and $\mathbb{R}^{2} / \Gamma_{e q}$ with $\Gamma_{e q}=\mathbf{Z}(1,0) \oplus$ $\mathbf{Z}(1 / 2, \sqrt{3} / 2)$. These two metrics are also the only extremal metrics for $\lambda_{1}$ (Corollary 2.2). Moreover, we prove that for each of them, the standard embedding (in $\mathbb{S}^{3}$ for $g_{c l}$ and $\mathbb{S}^{5}$ for $g_{e q}$ ) is, up to equivalence, the only full (minimal) isometric immersion by the first eigenfunctions.

Note that a first step towards this classification was achieved by Montiel and Ros [6] who proved that the only minimal torus immersed in $\mathbb{S}^{3}$ by its first eigenfunctions is the Clifford torus. They deduced that if the aforementioned conjecture of Yau is true, then the Clifford torus is the only minimally embedded torus in $\mathbb{S}^{3}$ (Lawson's conjecture).

\section{Extremal metrics for the $\lambda_{1}$ functional.}

Let $M$ be a compact smooth manifold of dimension $m \geq 2$. Denote by $\mathcal{R}_{0}(M)$ the set of Riemannian metrics of volume 1 on $M$. For any $g \in$ $\mathcal{R}_{0}(M)$, we denote by $0<\lambda_{1}(g) \leq \lambda_{2}(g) \leq \cdots \leq \lambda_{k}(g) \leq \cdots$ the increasing sequence of eigenvalues of the Laplacian $\Delta_{g}$ of $g$. The functional:

$$
\begin{array}{rll}
\lambda_{1} \quad: \quad \mathcal{R}_{0}(M) & \rightarrow & \mathbb{R} \\
g & \mapsto \lambda_{1}(g)
\end{array}
$$


is continuous but not differentiable in general. However, for any family $\left(g_{t}\right)_{t}$ of metrics, analytic in $t, \lambda_{1}\left(g_{t}\right)$ has right and left derivatives w.r.t. $t$. Indeed, if $\left(g_{t}\right)_{t \in]-\delta, \delta[}$ is such a family and if $k$ is the multiplicity of $\lambda_{1}\left(g_{0}\right)$, then there exists $k$ analytic families $\Lambda_{1, t}, \ldots, \Lambda_{k, t}$ of real numbers and $k$ analytic families of smooth functions $u_{1, t}, \ldots, u_{k, t}$ such that: $\forall i \leq k$ and $\forall t$, $\Delta_{g_{t}} u_{i, t}=\Lambda_{i, t} u_{i, t}, \Lambda_{i, 0}=\lambda_{1}\left(g_{0}\right)$ and $\left\{u_{1, t}, \ldots, u_{k, t}\right\}$ is $L_{2}\left(g_{t}\right)$-orthonormal (see [1] and [2] for details). Moreover, Berger [2] gave the following formula for the derivative of $\Lambda_{i, t}$ :

$$
\left.\frac{d}{d t} \Lambda_{i, t}\right|_{t=0}=-\int_{M}\left\langle q\left(u_{i}\right), h\right\rangle \nu_{g_{0}}
$$

where $\nu_{g_{0}}$ is the Riemannian volume element of $g_{0}, u_{i}=u_{i, 0}, h=\left.\frac{d}{d t} g_{t}\right|_{t=0}$, $\langle$,$\rangle is the inner product induced by g_{0}$ on the space $S^{2}(M)$ of symmetric covariant 2-tensors of $M$ and where for any $u \in C^{\infty}(M)$,

$$
q(u)=d u \otimes d u+\frac{1}{4} \Delta_{g_{0}}\left(u^{2}\right) g_{0} .
$$

From the continuity of $\lambda_{i}\left(g_{t}\right)$ and $\Lambda_{i, t}$ w.r.t. $t$, we have for $t$ small enough $\left\{\Lambda_{i, t}\right\}_{1 \leq i \leq k}=\left\{\lambda_{i}\left(g_{t}\right)\right\}_{1 \leq i \leq k}$ and thus $\lambda_{1}\left(g_{t}\right)=\min _{1 \leq i \leq k}\left\{\Lambda_{i, t}\right\}$. This proves the left and right differentiability of $\lambda_{1}\left(g_{t}\right)$ and gives:

$$
\left.\frac{d}{d t} \lambda_{1}\left(g_{t}\right)\right|_{t=0^{+}}=\left.\min _{1 \leq i \leq k} \frac{d}{d t} \Lambda_{i, t}\right|_{t=0}=-\max _{1 \leq i \leq k} \int_{M}\left\langle q\left(u_{i}\right), h\right\rangle \nu_{g_{0}},
$$

and

$$
\left.\frac{d}{d t} \lambda_{1}\left(g_{t}\right)\right|_{t=0^{-}}=\left.\max _{1 \leq i \leq k} \frac{d}{d t} \Lambda_{i, t}\right|_{t=0}=-\min _{1 \leq i \leq k} \int_{M}\left\langle q\left(u_{i}\right), h\right\rangle \nu_{g_{0}} .
$$

This suggests the following definition:

Definition 1.1. A metric $g \in \mathcal{R}_{0}(M)$ is said to be extremal for the $\lambda_{1}$ functional if for any analytic deformation $\left(g_{t}\right)_{t} \subset \mathcal{R}_{0}(M)$, with $g_{0}=g$, the left and right derivatives of $\lambda_{1}\left(g_{t}\right)$ at $t=0$ have opposite signs, i.e.,

$$
\left.\frac{d}{d t} \lambda_{1}\left(g_{t}\right)\right|_{t=0^{+}} \leq 0 \leq\left.\frac{d}{d t} \lambda_{1}\left(g_{t}\right)\right|_{t=0^{-}} .
$$

This last condition is equivalent to :

$$
\lambda_{1}\left(g_{t}\right) \leq \lambda_{1}(g)+o(t) \text { as } t \rightarrow 0 .
$$

Hence our definition of extremality is a equivalent formulation of Nadirashvili's one [7].

The main result of this section is:

Theorem 1.1. If a Riemannian metric $g \in \mathcal{R}_{0}(M)$ is extremal for $\lambda_{1}$ then it is $\lambda_{1}$-minimal. 
In the 2-dimensional case this result was proved by Nadirashvili [7]. Some of the arguments in our proof are inspired by his. However, the use of the aforementioned result of Berger makes the proof of this theorem simpler and more transparent.

Lemma 1.1. If a metric $g \in \mathcal{R}_{0}(M)$ is extremal for $\lambda_{1}$ then for any $h \in$ $S_{0}^{2}(M)=\left\{h \in S^{2}(M) ; \int_{M} t r_{g} h \nu_{g}=0\right\}$ there exists $u \in E_{1}(g) \backslash\{0\}$ such that:

$$
\int_{M}\langle q(u), h\rangle \nu_{g}=0
$$

Proof. Suppose that $g$ is extremal for $\lambda_{1}$ and let $h \in S_{0}^{2}(M)$. We let, for small $t, g_{t}=\frac{g+t h}{V(g+t h)^{2 / m}} \in \mathcal{R}_{0}(M)$, where $V(g+t h)$ is the Riemannian volume of $g+t h$. Since $\left.\frac{d}{d t} V(g+t h)\right|_{t=0}=\frac{1}{2} \int_{M} t r_{g} h \nu_{g}=0$, we find $\left.\frac{d}{d t} g_{t}\right|_{t=0}=h$. The extremality condition implies that the quadratic form $u \in E_{1}(g) \mapsto \int_{M}\langle q(u), h\rangle \nu_{g}$ takes on both nonpositive and nonnegative values, and therefore it admits at least one isotropic direction.

Proof of Theorem 1.1. Let $K$ be the convex hull in $S^{2}(M)$ of $\{q(u), u \in$ $\left.E_{1}(g)\right\}$. The set $K \cup\{g\}$ is contained in a finite dimensional subspace of $S^{2}(M)$. We claim that $g \in K$. Indeed, if $g \notin K$ then, since $K$ is a convex cone, the Hahn-Banach theorem implies the existence of $s \in S^{2}(M)$ such that:

$$
\int_{M}\langle s, g\rangle \nu_{g}>0 \text { and for every } l \in K \backslash\{0\}, \int_{M}\langle l, s\rangle \nu_{g}<0 \text {. }
$$

The 2-tensor $\tilde{s}=s-\frac{\left(\int_{M}\langle s, g\rangle \nu_{g}\right)}{m V(g)} g$ belongs to $S_{0}^{2}(M)$ and, for any $u \in$ $E_{1}(g) \backslash\{0\}$,

$$
\begin{aligned}
& \int_{M}\langle q(u), \tilde{s}\rangle \nu_{g} \\
& =\int_{M}\langle q(u), s\rangle \nu_{g}-\frac{1}{m V(g)}\left(\int_{M}\langle s, g\rangle \nu_{g}\right)\left(\int_{M}|d u|^{2} \nu_{g}\right)<0 .
\end{aligned}
$$

By Lemma 1.1, this contradicts the extremality of $g$.

Thus $g \in K$ and there exists $w_{1}, \ldots w_{d} \in E_{1}(g)$ such that:

$$
\begin{aligned}
g & =\sum_{1 \leq i \leq d} q\left(w_{i}\right)=\sum_{1 \leq i \leq d} d w_{i} \otimes d w_{i}+\frac{1}{4}\left(\sum_{1 \leq i \leq d} \Delta w_{i}^{2}\right) g \\
& =\sum_{1 \leq i \leq d}\left(d w_{i} \otimes d w_{i}+\frac{1}{2}\left(\lambda_{1}(g) w_{i}^{2}-\left|d w_{i}\right|^{2}\right) g\right) .
\end{aligned}
$$


The traceless part of the last member of this equation must be zero. Therefore,

$$
\sum_{1 \leq i \leq d}\left(d w_{i} \otimes d w_{i}-\frac{\left|d w_{i}\right|^{2}}{m} g\right)=0
$$

and then:

$$
\frac{\lambda_{1}}{2} \sum_{1 \leq i \leq d} w_{i}^{2}=1+\left(\frac{m-2}{2 m}\right) \sum_{1 \leq i \leq d}\left|d w_{i}\right|^{2} .
$$

The $\lambda_{1}$-minimality of $g$ will follow from the fact that $\sum_{1 \leq i \leq d}\left|d w_{i}\right|^{2}$ is constant and equal to $m$. Indeed, set $f=\left(\sum_{1 \leq i \leq d} w_{i}^{2}\right)-\frac{m}{\lambda_{1}(g)}$. From (1) we get:

$$
(m-2) \Delta_{g} f=2(m-2)\left(\lambda_{1}(g)\left(\sum_{1 \leq i \leq d} w_{i}^{2}\right)-\sum_{1 \leq i \leq d}\left|d w_{i}\right|^{2}\right)=-4 \lambda_{1}(g) f .
$$

This implies that $f=0$ (the Laplacian being a positive operator). Therefore $\left(\sum_{1 \leq i \leq d} w_{i}^{2}\right)=\frac{m}{\lambda_{1}(g)}$. Replacing in (1) we obtain $\sum_{1 \leq i \leq d}\left|d w_{i}\right|^{2}=m$.

In [4] we showed that $\lambda_{1}$-minimal metrics satisfy certain remarkable conformal properties. Theorem 1.1 tells us that all these properties are still true for extremal metrics:

Corollary 1.1. Let $g \in \mathcal{R}_{0}(M)$ be an extremal metric for $\lambda_{1}$.

(i) The multiplicity of $\lambda_{1}(g)$ satisfies: $\operatorname{mult}\left(\lambda_{1}(g)\right) \geq m+1$, where equality holds if and only if $g$ is isometric to a standard metric of a Euclidean sphere.

(ii) For any $g^{\prime} \in C_{0}(g)=\left\{g^{\prime} \in \mathcal{R}_{0}(M) ; g^{\prime}\right.$ conformal to $\left.g\right\}$ we have $\lambda_{1}\left(g^{\prime}\right) \leq \lambda_{1}(g)$, and equality holds if and only if $g^{\prime}$ is isometric to $g$. In particular, the functional $\lambda_{1}$ does not admit a local minimum in $\mathcal{R}_{0}(M)$.

(iii) The metric $g$ is, up to isometry, the only extremal metric of $\lambda_{1}$ in $C_{0}(g)$.

(iv) If $(M, g)$ is not isometric to a Euclidean sphere then any conformal diffeomorphism of $(M, g)$ is an isometry.

The following is a converse to Theorem 1.1.

Proposition 1.1. Let $g \in \mathcal{R}_{0}(M)$ and assume there exists an $L_{2}(g)$-orthonormal basis $\left\{\phi_{1}, \ldots, \phi_{k}\right\}$ of $E_{1}(g)$ such that the 2 -tensor $\sum_{1 \leq i \leq k} d \phi_{i} \otimes d \phi_{i}$ is proportional to $g$. Then $g$ is extremal for $\lambda_{1}$. 
Proof. Let $\left(g_{t}\right)_{t} \subset \mathcal{R}_{0}(M)$ be a family of metrics analytic in $t$ with $g_{0}=g$ and set $h=\left.\frac{d}{d t} g_{t}\right|_{t=0}$. With the same notation as above we have for small $t$ :

$$
\sum_{1 \leq i \leq k} \lambda_{i}\left(g_{t}\right)=\sum_{1 \leq i \leq k} \Lambda_{i, t}
$$

Therefore, $\sum_{1 \leq i \leq k} \lambda_{i}\left(g_{t}\right)$ is differentiable at $t=0$ and

$$
\left.\frac{d}{d t} \sum_{1 \leq i \leq k} \lambda_{i}\left(g_{t}\right)\right|_{t=0}=\left.\frac{d}{d t} \sum_{1 \leq i \leq k} \Lambda_{i, t}\right|_{t=0}=\operatorname{trace} Q_{h},
$$

where $Q_{h}$ is the quadratic form defined on $E_{1}(g)$ by:

$$
Q_{h}(u)=\int_{M}\langle q(u), h\rangle \nu_{g}
$$

and where the trace of $Q_{h}$ is taken w.r.t the $L_{2}$ inner product induced by $g$. Now

$$
\begin{aligned}
\operatorname{trace} Q_{h} & =\sum_{1 \leq i \leq k} Q_{h}\left(\phi_{i}\right) \\
& =\int_{M}\left\langle\sum_{1 \leq i \leq k} d \phi_{i} \otimes d \phi_{i}, h\right\rangle \nu_{g}+\frac{1}{4} \sum_{1 \leq i \leq k} \int_{M}\left\langle\Delta \phi_{i}^{2}, h\right\rangle \nu_{g} .
\end{aligned}
$$

Since $\sum_{1 \leq i \leq k} d \phi_{i} \otimes d \phi_{i}$ is proportional to $g$ and $\int_{M}\langle g, h\rangle \nu_{g}=\left.2 \frac{d}{d t} V\left(g_{t}\right)\right|_{t=0}=$ 0 we have $\int_{M}\left\langle\sum_{1 \leq i \leq k} d \phi_{i} \otimes d \phi_{i}, h\right\rangle \nu_{g}=0$. Moreover, by Takahashi's theorem $\sum_{1 \leq i \leq k} \phi_{i}^{2}$ is constant. Therefore, trace $Q_{h}=0$ and $\left.\frac{d}{d t} \sum_{1 \leq i \leq k} \lambda_{i}\left(g_{t}\right)\right|_{t=0}$ $=0$. The extremality of $g$ then follows from the inequality $\lambda_{1}\left(g_{t}\right) \stackrel{t=0}{\leq}$ $\frac{1}{k} \sum_{1 \leq i \leq k} \lambda_{i}\left(g_{t}\right)$ which is an equality at $t=0$.

\section{Remarks.}

1) It is known that compact irreducible homogeneous Riemannian spaces satisfy the hypothesis of Proposition 1.1 (see [8]). Thus, their standard metrics are extremal for $\lambda_{1}$.

2) We restricted ourselves to $\lambda_{1}$. Nevertheless, the results of this paragraph can be carried over to the case of higher eigenvalues.

\section{2. $\lambda_{1}$-minimal and extremal metrics on the torus.}

Let $(M, g)$ be an orientable compact surface of genus one endowed with a Riemannian metric $g$. It is well known that there exists a lattice $\Gamma$ of $\mathbb{R}^{2}$ such that $(M, g)$ is conformally equivalent to the torus $\left(\mathbb{R}^{2} / \Gamma, g_{\Gamma}\right)$, where $g_{\Gamma}$ is the flat metric induced from the Euclidean metric on $\mathbb{R}^{2}$. The Clifford torus $\left(\mathbf{T}_{c l}^{2}=\mathbb{R}^{2} / \Gamma_{c l}, g_{c l}=g_{\Gamma_{c l}}\right)$ with $\Gamma_{c l}=\mathbf{Z}(1,0) \oplus \mathbf{Z}(0,1)$, and the equilateral torus $\left(\mathbf{T}_{e q}^{2}=\mathbb{R}^{2} / \Gamma_{e q}, g_{e q}=g_{\Gamma_{e q}}\right)$ with $\Gamma_{e q}=\mathbf{Z}(1,0) \oplus \mathbf{Z}(1 / 2, \sqrt{3} / 2)$, 
each admit a natural homothetic minimal embedding into a sphere. These embeddings, denoted by $\phi_{c l}$ and $\phi_{e q}$, are those induced on $\mathbf{T}_{c l}^{2}$ and $\mathbf{T}_{e q}^{2}$ from $\tilde{\phi}_{c l}: \mathbb{R}^{2} \rightarrow \mathbb{S}^{3}$, where $\tilde{\phi}_{c l}(x, y)=\frac{1}{\sqrt{2}}(\exp 2 i \pi x, \exp 2 i \pi y)$, and $\tilde{\phi}_{e q}: \mathbb{R}^{2} \rightarrow \mathbb{S}^{5}$, where $\tilde{\phi}_{e q}(x, y)=\frac{1}{\sqrt{3}}(\exp 4 i \pi y / \sqrt{3}, \exp 2 i \pi(x-y / \sqrt{3}), \exp 2 i \pi(x+y / \sqrt{3}))$.

Theorem 2.1. Let $(M, g)$ be a compact orientable surface of genus one and suppose that there exists a full isometric immersion $\phi=\left(\phi_{1}, \ldots, \phi_{n+1}\right)$ from $(M, g)$ in the $n$-dimensional unit sphere $\mathbb{S}^{n}$ such that $\forall i \leq n+1, \phi_{i} \in E_{1}(g)$. Then either:

(i) $(M, g)$ is isometric to the normalized Clifford torus $\left(\mathbf{T}_{c l}^{2}, 2 \pi^{2} g_{c l}\right), n=3$ and $\phi$ is equivalent to $\phi_{c l}$, or

(ii) $(M, g)$ is isometric to the normalized equilateral torus $\left(\mathbf{T}_{e q}^{2}, \frac{8 \pi^{2}}{3} g_{e q}\right)$, $n=5$ and $\phi$ is equivalent to $\phi_{e q}$.

Recall that an immersion $\phi$ into $\mathbb{S}^{n}$ is full if its image is not contained in a great sphere of $\mathbb{S}^{n}$. Two immersions $\phi$ and $\psi$ into $\mathbb{S}^{n}$ are called equivalent if there exists an isometry $R$ of $\mathbb{S}^{n}$ such that $\phi=R \circ \psi$. A direct consequence of Theorem 2.1 is:

Corollary 2.1. A compact genus one orientable surface $(M, g)$ is $\lambda_{1}$-minimal if and only if it is homothetic to $\left(\mathbf{T}_{c l}^{2}, g_{c l}\right)$ or $\left(\mathbf{T}_{e q}^{2}, g_{e q}\right)$.

As the metrics $g_{c l}$ and $g_{e q}$ trivially satisfy the hypothesis of Proposition 1.1 we have the following:

Corollary 2.2. Let $M$ be a compact orientable surface of genus one. A metric $g$ on $M$ is extremal for $\lambda_{1}$ if and only if $(M, g)$ is homothetic to $\left(\mathbf{T}_{c l}^{2}, g_{c l}\right)$ or $\left(\mathbf{T}_{e q}^{2}, g_{e q}\right)$.

The proof of Theorem 2.1 is based on the following Propositions 2.1 and 2.2 which are valid in a more general setting.

Proposition 2.1. Let $(M, g)$ be a n-dimensional compact Riemannian homogeneous manifold non homothetic to $\mathbb{S}^{n}$. If a metric $g=f g_{0}$, conformal to $g_{0}$, is $\lambda_{1}$-minimal, then $f$ is constant on $M$.

Proof. As $(M, g)$ is $\lambda_{1}$-minimal non homothetic to $\mathbb{S}^{n}$ then any conformal diffeomorphism of $(M, g)$ is an isometry (cf. [4]). It follows that any isometry of $\left(M, g_{0}\right)$ is also an isometry of $(M, g)$. Thus the function $f$ is invariant under the isometry group of $\left(M, g_{0}\right)$. The result follows from the homogeneity of $\left(M, g_{0}\right)$.

Proposition 2.2. Let $\eta_{1}, \eta_{2}, \ldots, \eta_{N}$ be $N$ continuous functions on a domain $\Omega$ of $\mathbb{R}^{m}$ and assume that the $N^{2}$ functions: $2 \eta_{j}(1 \leq j \leq N), \eta_{k}+\eta_{l}$ and $\eta_{k}-\eta_{l}(1 \leq k<l \leq N)$ are non-constant and mutually distinct modulo $2 \pi$. If $\phi=\left(\phi_{1}, \ldots, \phi_{n+1}\right)$ is a map from $\Omega$ to $\mathbb{S}^{n}$ such that all its components $\phi_{i}$ are in the vector space generated by $\left\{\cos \eta_{j}, \sin \eta_{j}, 1 \leq j \leq N\right\}$, 
then there exists an isometry $R$ of $\mathbb{S}^{n}$ such that

$$
R \circ \phi=\left(\alpha_{1} \exp i \eta_{j_{1}}, \alpha_{2} \exp i \eta_{j_{2}}, \ldots, \alpha_{r} \exp i \eta_{j_{r}}, 0, \ldots, 0\right),
$$

where $r \leq(n+1) / 2, j_{1}, \ldots, j_{r} \in\{1, \ldots, N\}$ and $\alpha_{1}, \ldots, \alpha_{r}$ are positive constants satisfying $\sum_{1 \leq j \leq r} \alpha_{j}^{2}=1$. In particular, $R(\phi(\Omega)) \subset \mathbb{S}^{1}\left(\alpha_{1}\right) \times$ $\mathbb{S}^{1}\left(\alpha_{2}\right) \times \cdots \times \mathbb{S}^{1}\left(\alpha_{r}\right) \times\{0\}$.

The proof of this proposition is quite elementary and can be omitted.

Proof of Theorem 2.1. In view of Proposition 2.1, it suffices to consider the case where the metric $g$ is flat. It is well known that there exists $(a, b) \in \mathbb{R}^{2}$; $0 \leq a \leq \frac{1}{2}, b>0$ and $a^{2}+b^{2} \geq 1$, such that $(M, g)$ is homothetic to $\left(\mathbf{T}_{a, b}^{2}=\mathbb{R}^{2} / \Gamma(a, b), g_{a b}=g_{\Gamma(a, b)}\right)$ with $\Gamma(a, b)=\mathbf{Z}(1,0) \oplus \mathbf{Z}(a, b)$ (cf. [3]). Now the existence of an isometric immersion from $(M, g)$ into the unit sphere by the first eigenfunctions implies that $\lambda_{1}(g)=2$. Since $\lambda_{1}\left(g_{a b}\right)=\frac{4 \pi^{2}}{b^{2}}$, $(M, g)$ is in fact isometric to $\left(\mathbf{T}_{a, b}^{2}, \frac{2 \pi^{2}}{b^{2}} g_{a b}\right)$. Let $E_{a, b}$ be the first eigenspace of $g_{a b}$ and $\phi:\left(\mathbf{T}_{a, b}^{2}, \frac{2 \pi^{2}}{b^{2}} g_{a b}\right) \rightarrow \mathbb{S}^{n}$ a full isometric immersion whose components $\phi_{i} \in E_{a, b}$.

- If $a^{2}+b^{2}>1$ then the dimension of $E_{a, b}$ is 2 and there is no such $\phi$.

- If $a^{2}+b^{2}=1$ and $(a, b) \neq(1 / 2, \sqrt{3} / 2)$ then $E_{a, b}$ is generated by $\cos \eta_{j}$, $\sin \eta_{j}, j \leq 2$, with $\eta_{1}(x, y)=\frac{2 \pi y}{b}$ and $\eta_{2}(x, y)=2 \pi\left(x-\frac{a y}{b}\right)$. From Proposition 2.2, it follows that $n=3$ and, up to an isometry of $\mathbb{S}^{3}$, $\phi$ has the form $\phi=\left(\alpha_{1} \exp \left(i \eta_{1}\right), \alpha_{2} \exp \left(i \eta_{2}\right)\right)$ with $\alpha_{1}>0, \alpha_{2}>0$ and $\alpha_{1}^{2}+\alpha_{2}^{2}=1$. As $\phi$ is isometric we deduce that $a=0, b=1$ and $\alpha_{1}=\alpha_{2}=\sqrt{2} / 2$. Thus $(M, g)$ is isometric to $\left(\mathbf{T}_{c l}^{2}, 2 \pi^{2} g_{c l}\right)$ and $\phi$ is equivalent to $\phi_{c l}$.

- If $(a, b)=(1 / 2, \sqrt{3} / 2)$ then $E_{a, b}$ is generated by $\cos \eta_{j}, \sin \eta_{j}, j \leq 3$, where $\eta_{1}(x, y)=4 \pi y / \sqrt{3}, \eta_{2}(x, y)=2 \pi\left(x-\frac{y}{\sqrt{3}}\right)$ and $\eta_{3}(x, y)=$ $2 \pi\left(x+\frac{y}{\sqrt{3}}\right)$. As before $n \leq 5$ and, up to isometry, $\phi=\left(\alpha_{1} \exp \left(i \eta_{1}\right)\right.$, $\left.\alpha_{2} \exp \left(i \eta_{2}\right), \alpha_{3} \exp \left(i \eta_{3}\right)\right)$ where $\alpha_{1}, \alpha_{2}$ and $\alpha_{3}$ are nonnegative constants such that $\alpha_{1}^{2}+\alpha_{2}^{2}+\alpha_{3}^{2}=1$. As $\phi$ is isometric we obtain $\alpha_{1}=\alpha_{2}=\alpha_{3}=\sqrt{3} / 3$. Thus $\phi$ is equivalent to $\phi_{e q}$.

\section{References}

[1] S. Bando and H. Urakawa, Generic properties of the eigenvalue of Laplacian for compact Riemannian manifolds, Tôhoku Math. J., 35 (1983), 155-172.

[2] M. Berger, Sur les premières valeurs propres des variétés riemanniennes, Compositio. Math., 26 (1973), 129-149. 
[3] M. Berger, P. Gauduchon and E. Mazet, Le spectre d'une variété riemannienne, Lecture Notes in Math., Vol. 194, Springer, 1971.

[4] A. El Soufi and S. Ilias, Immersions minimales, première valeur propre du laplacien et volume conforme, Math. Ann., 275 (1986), 257-267.

[5] __ Majoration de la seconde valeur propre d'un opérateur de Schrø̈dinger sur une variété compacte et applications, J. of Funct. Anal., 103(2) (1992), 294-316.

[6] S. Montiel and A. Ros, Minimal immersions of surfaces by the first eigenfunctions and conformal area, Invent. Math., 83 (1986), 153-166.

[7] N. Nadirashvili, Berger's isoperimetric problem and minimal immersions of surfaces, Geom. and Funct. Anal., 6 (1996), 877-897.

[8] T. Takahashi, Minimal immersions of riemannian manifolds, J. Math. Soc. Jap., 18 (1966), 380-385.

[9] S.-T. Yau, Problems section. Seminar on Differential Geometry, Ann. Math. Stud., Vol. 102, Princeton University Press, 1982.

Received October 20, 1998 and revised June 15, 1999.

LABORATOIRE DE MATHEMATIQUES ET PHYSIQUE THEORIQUE

UNIVERSITE DE TOURS

PARC DE GRandmont

37200 TOURS

FRANCE

E-mail address: elsoufi@univ-tours.fr

LABORATOIRE DE MATHEMATIQUES ET PHYSIQUE THEORIQUE

UNIVERSITE DE TOURS

PARC DE GRANDMONT

37200 TOURS

FRANCE

E-mail address: ilias@univ-tours.fr 